\title{
Medical Evacuation: Im Krisengebiet eine besondere Herausforderung
}

\section{J. Langegger}

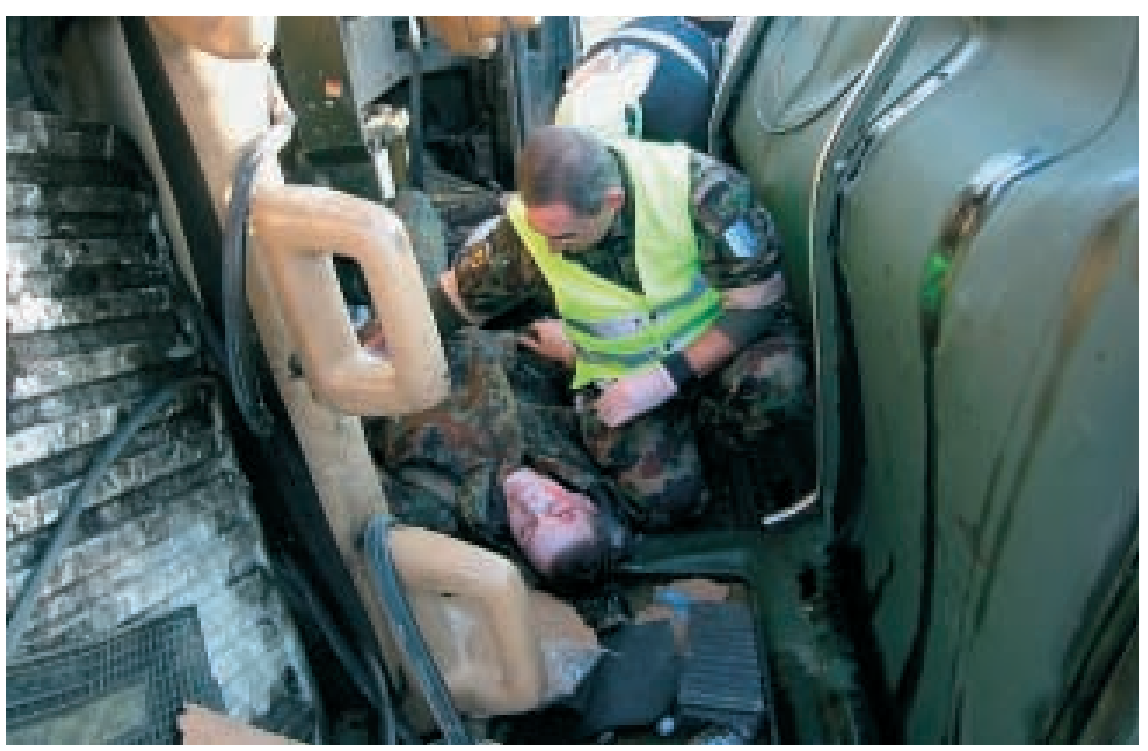

«Ein Patient mit Polytrauma und ein Patient mit Splitterverletzungen. Wir brauchen ein Sanitätsfahrzeug, ein Minenräumungsteam und die Feuerwehr!» So tönt es im Stationszimmer aus dem Funk. Zum Glück handelt es sich nur um eine Übung: Im südlichen Kosovo liegt das Militärcamp «Casablanca» der Swisscoy (schweizerische Beteiligung der KFOR). Diese leistet seit 1999, seit dem Ende des Kriegs, ihren Einsatz für die Friedenssicherung. Rund 200 Schweizerinnen und Schweizer arbeiten hier jeweils sechs Monate lang in diversen Bereichen, unter anderem im Medical Team, das sich aus zwei Ärztinnen/Ärzten und momentan vier Krankenschwestern zusammensetzt. Dieses Team gewährleistet rund um die Uhr die medizinische Versorgung der Soldaten. Es arbeitet im Rahmen einer multinationalen Brigade zusammen mit einer deutschen und einer österreichischen Belegschaft.

\section{Bergung mitten im Minenfeld}

Korrespondenz: Julia Langegger PIO Press \& Information Officers Swisscoy 9 CH-00599 Militär
Wenn Verletzte unter erschwerten Bedingungen, also in einem von Krieg und Krisen gezeichneten Land, geborgen werden müssen, stellen sich viele zusätzliche Fragen. In einem militärischen Umfeld gibt es zudem rigide Sicherheitsauflagen.
Damit ist beispielsweise gemeint, dass militärische Fahrzeuge nicht unbeaufsichtigt herumstehen dürfen oder dass nach der persönlichen Waffe, die ein verunfallter Soldat verloren hat, gesucht werden muss.

Im Kosovo gibt es immer noch viele schlechte Strassen, die übersät sind mit zahlreichen tiefen Schlaglöchern. Dies erschwert den Patiententransport. Blaulicht und Zweiklanghorn bedeuten zudem nicht in jedem Land so wie in der Schweiz, dass andere Fahrzeuge dem Rettungswagen auch tatsächlich Platz machen. Die Fahrweise der Kosovaren ist abgesehen davon teilweise äusserst bedenklich. Es ist landesweit üblich, an den unübersichtlichsten Stellen waghalsig zu überholen und mit erhöhter Geschwindigkeit durch Ortschaften zu brausen.

$\mathrm{Zu}$ den schlechten Strassenverhältnissen kommt nach wie vor die Minengefahr hinzu. Befinden sich die in Not geratenen Personen in einem minenverseuchten Gebiet, müssen die Minenspezialisten alarmiert werden. Hier prallen möglicherweise militärische Sicherheitsauflagen und zivile Strukturen aufeinander. Beispielsweise sieht die lokale Bevölkerung in einer Wiese, die sie selbst schon oft betreten hat, keine Gefahr. Ist das Areal aber nicht mit einer 100prozentigen Sicherheit minenfrei, so muss es zuerst von Spezialisten inspiziert und gesichert werden, bevor das militärische Rettungsteam zu den Verletzten gelangt. Weiter kompliziert die Rettungsarbeit, dass das Material (Standard der Funkgeräte, Tragbahren, Fahrzeuge, Platzverhältnisse usw.) teilweise nicht mehr neuwertig ist und darum nicht immer zuverlässig funktioniert.

Gerade deshalb sind Übungen für ein multinationales Rettungsteam so wichtig: Es gilt dabei, Mängel festzustellen, um diese für den Ernstfall zu beheben. Alle Beteiligten können hiervon profitieren, dazulernen und ihr persönliches Fachwissen erweitern.

\section{Übung macht den Meister}

Die medizinische Belegschaft des Camp Casablanca ist glücklicherweise zu 95\% mit Grippeviren, Magen-Darm-Erregern oder verstauchten 

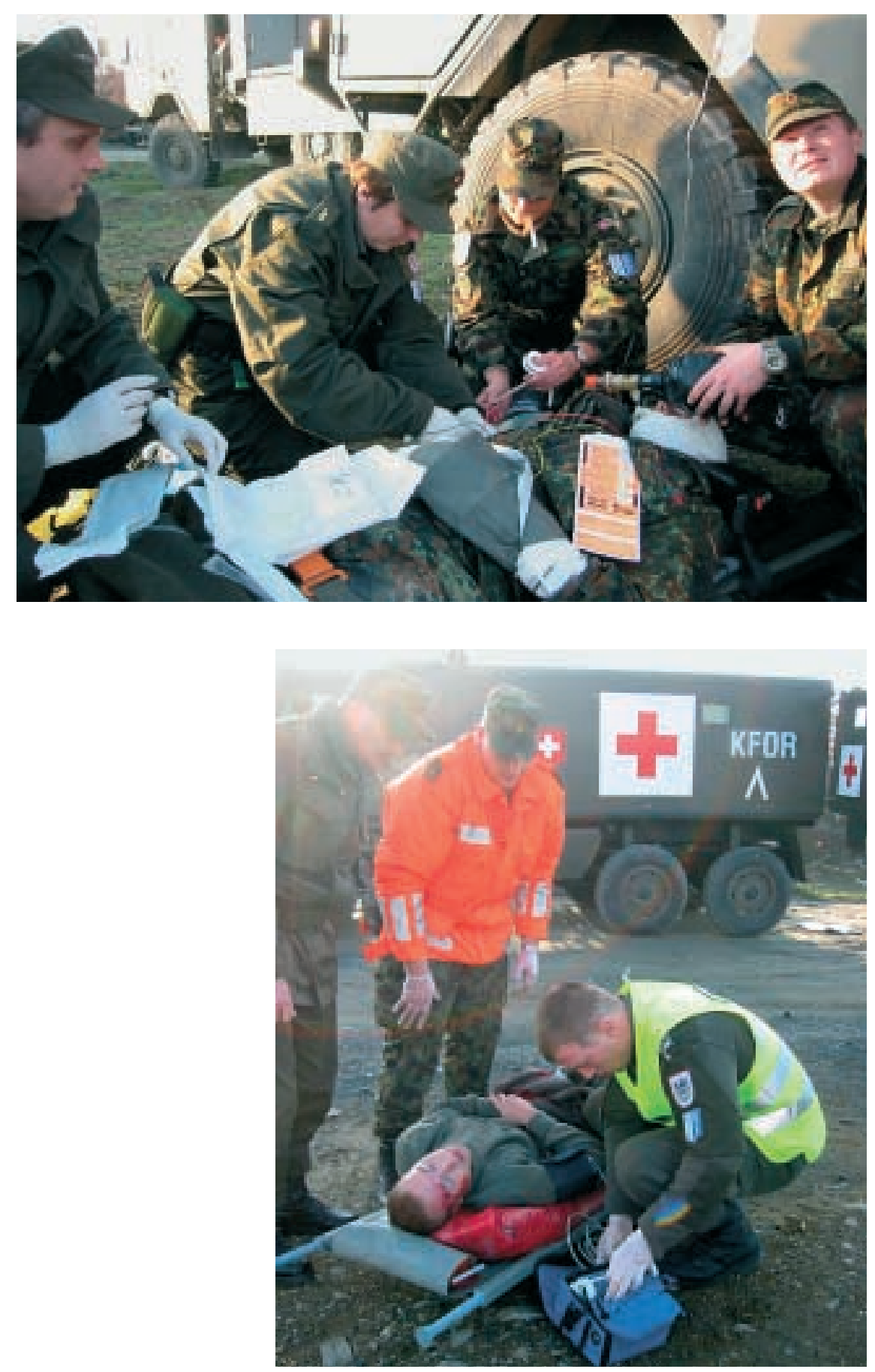

Knöcheln konfrontiert. Das Team wendet die meiste Zeit für relativ harmlose Fälle auf, muss aber im Ernstfall innert fünf Minuten einsatzbereit sein. Übungen helfen, dem Notfall etwas «näherzukommen». Viele der medizinischen Mitarbeiter, die im Rahmen des KFOR-Einsatzes arbeiten, haben wenig Notfallerfahrung, dies trifft auch für manche Ärztinnen und Ärzte zu. Also heisst es hier unten im Kosovo: üben, üben, üben. Gerade im Bereich Medical Evacuation gibt es immer Handgriffe und Abläufe, die zu optimieren sind.

\section{Vorbereitungen für das Evakuationstraining}

Die Übung wird durchgeführt mit einem schweizerischen, einem deutschen und einem österreichischen Team. Dazu kommen die Minenspezialisten und Figuranten sowie zahlreiche Feuerwehrleute. Allesamt sind sie Angehörige ihrer nationalen Einheiten für die Friedensförderung. Dazu kommt eine grosse Anzahl von Notfahrzeugen aller Art.

An jenem Morgen ist der Sanitätsbereich des Camps von Hektik erfüllt. Die Koordination einer solchen Übung ist kein leichtes. Die Figuranten müssen als Vorhut an den Unfallorten ihre Plätze beziehen. Die Fahrzeuge sind abfahrbereit, das Material komplett; die Rettungsrucksäcke, die stolze $20 \mathrm{~kg}$ wiegen, sind gemäss Checklisten vollständig und stehen bereit.

Nach dem Briefing der Teams ist es dann soweit. Alle Vorbereitungen sind getroffen. Die Figuranten wissen, was sich wo abspielen wird und wer auf welchem Fahrzeug mitfährt. Sie sind geschminkt mit künstlichem Blut, und die mit Kunststoffmasse für Moulagen geformten «Knochenbrüche» sehen echt aus.

Mit einiger Enttäuschung erfahren wir, dass der amerikanische Helikopter vom Typ «Black Hawk» nicht an der Übung teilnehmen kann. Der Nebel über dem südlichen Kosovo ist an diesem Morgen schlichtweg zu dicht. Schade, denn ein «Spiderman», der sich aus dem Rumpf des Helikopters abseilt, um Verletzte aus unwegsamem oder vermintem Gebiet zu bergen, indem er sie mit sich hochzieht, ist kein alltägliches Bild und hätte die Übung noch mit zusätzlicher Spannung versehen. Es hat aber auch Vorteile, dass der Hubschrauber wetterbedingt nicht fliegen darf: Auch im Ernstfall kann es durchaus sein, dass das Wetter es nicht erlaubt, Hilfe aus der Luft anzufordern.

\section{Zwei Unfälle - viele Verletzte}

Durchgespielt werden zwei Szenarien: Auf dem ersten Schauplatz fährt ein Radschützenpanzer der österreichischen Armee, ein sogenannter Pandur, auf eine Mine auf und kommt in einem Minenfeld zum Stehen. Zwei Personen werden verletzt, ein Soldat erleidet Splitterverletzungen im Gesicht, Rippenserienfrakturen und einen offenen Bruch an der rechten Hand. Der andere hat ebenfalls Splitterverletzungen im Gesicht und einen psychogenen Schock.

Am zweiten Unfallort prallen zwei Kleinbusse abseits der Strasse ineinander. Dies verur- 


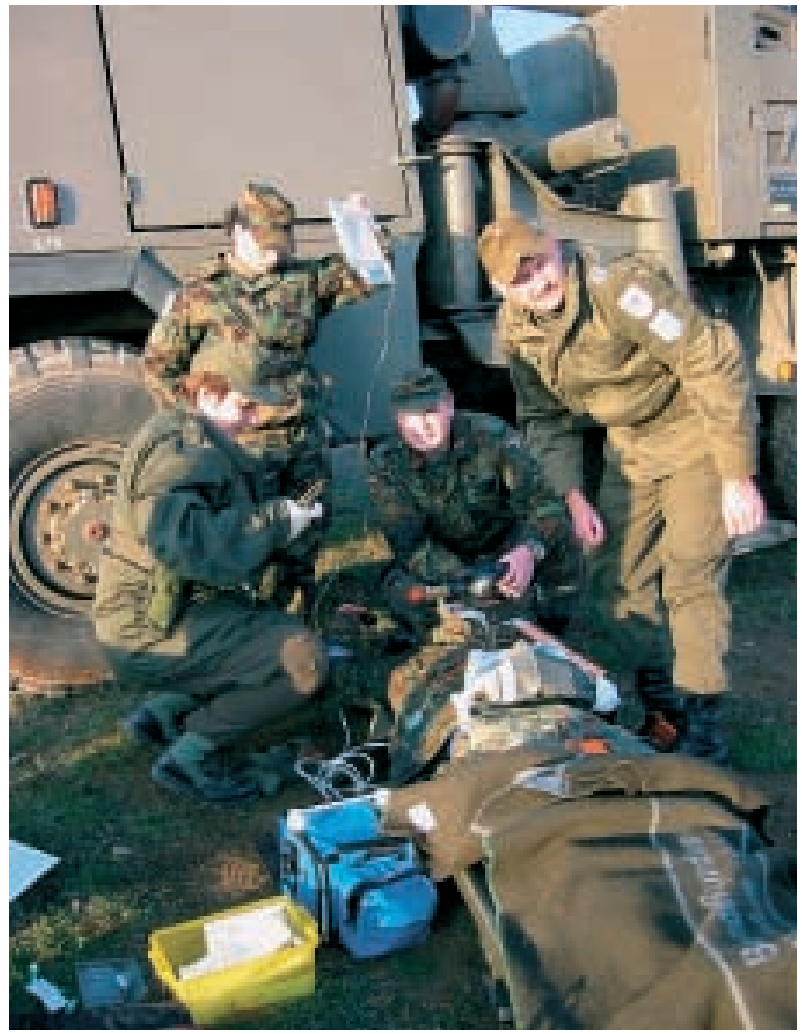

sacht gleich sechs Verletzte mit sehr unterschiedlichen Verletzungsmustern: Frakturen an Ober- und Unterschenkeln, ein stumpfes Abdominaltrauma, eine Verletzung im Bereich LWK 3-5 mit Sensibilitätsstörungen in beiden Beinen, ein Polytrauma, Verbrennungen an Oberkörper und Gesicht, Atmungsstörungen sowie Schnittverletzungen an den Armen.

\section{Durchspielen von Bergungsarbeit und Minimalversorgung}

Bei der Hinfahrt zu den Unfallorten haben die Figuranten noch gescherzt, doch sobald sie in den zerbeulten Fahrzeugen ihre Plätze einnehmen und die Rettungsfahrzeuge mit Blaulicht und Sirenengeheul eintreffen, nachdem $18 \mathrm{Mi}$ nuten zuvor der Notruf per Funk in der Zentrale eingegangen ist, vergeht auch ihnen ob der Echtheit der Situation das Lachen. Die Türen der Fahrzeuge springen auf, die Rettungsmannschaft organisiert sich blitzschnell. Unverzichtbar ist eine Person, die Anweisungen gibt, die die sogenannte Triage macht. Der Schweizer Arzt verschafft sich schnell einen Überblick und ordnet an. Zuviel Selbstinitiative ist fehl am Platz. Wer funkt? Wer sichert die Strasse ab? Braucht es Minenspezialisten? Welche Verletzten werden zuerst, welche zuletzt behandelt? Die Feuer- wehrleute sprechen sich ab, wer welche Bergung übernimmt. Sie werden benötigt, weil es am Unfallort brennt und Verletzte in Fahrzeugen eingeklemmt sind.

Der Unfallort wirkt so echt, dass die Lokalbevölkerung, die auf dem Feldweg mit ihren Autos unterwegs ist, einen Moment lang betroffen stehenbleibt - bis sie entdeckt, dass am Rande des Geschehens zwei Soldaten schmunzelnd eine Zigarette rauchen.

Nach ungefähr anderthalb Stunden sind die Verwundeten erstversorgt, eine Thorax-Drainage ist erfolgreich gesetzt. Es ist abgesprochen, welche der Verletzten in welche Krankenhäuser gebracht werden. Die Schwerverletzten sind abtransportiert und es sind genügend Rettungsfahrzeuge auf Platz für die Leichtverletzten. Zu guter Letzt werden auch die zerbeulten Kleinbusse wieder per Kran auf Lastwagen gehoben und ins Camp zurückgefahren.

\section{Debriefing und Fazit}

Das Debriefing dient dazu, alle Beteiligten zu Wort kommen zu lassen und die Probleme der Evakuation zu besprechen. Nur so können Rettungsabläufe optimiert werden. «Die richtigen Materialien müssen dabei sein - und waren auch dabei», so eine österreichische Ärztin. Sie zeigt sich zufrieden über den Ablauf der Übung. Auch die schweizerische Beteiligung und der deutsche Anteil äussern sich hauptsächlich positiv. Jede und jeder wusste, wo und in welcher Reihenfolge geholfen werden musste und wer welche Aufgaben übernehmen sollte. Dabei ist wichtig, dass die Person, die das Rettungsmanöver leitet, sowohl die Stärken als auch die Schwächen der Mitarbeitenden kennt und diese dementsprechend einteilt.

Die Handgriffe des Rettungspersonals sitzen. Bei den anderen Helferinnen und Helfern, die mit Notfallsituationen wenig vertraut sind, muss dies noch besser eingeübt werden. Beanstandet wird die Qualität der Funkgeräte, mit denen die Fahrzeuge ausgestattet sind, sie seien alt und deshalb unzuverlässig. Das ist jedoch im Einsatzland generell ein Problem: Die Geräte sind in vielen Bereichen nicht auf dem neuesten Stand. Dennoch, so lautet das Fazit, wird gute Arbeit geleistet - ein Kompliment an die Equipe, die flexibel sein muss. Fehlt ein Gerät oder ist es unzuverlässig, sucht man blitzschnell nach anderen Lösungen.

Teilweise bestehen, obwohl es sich um drei deutschsprachige Nationen handelt, doch auch Sprachbarrieren. Der Schweizer Arzt, der aus der 


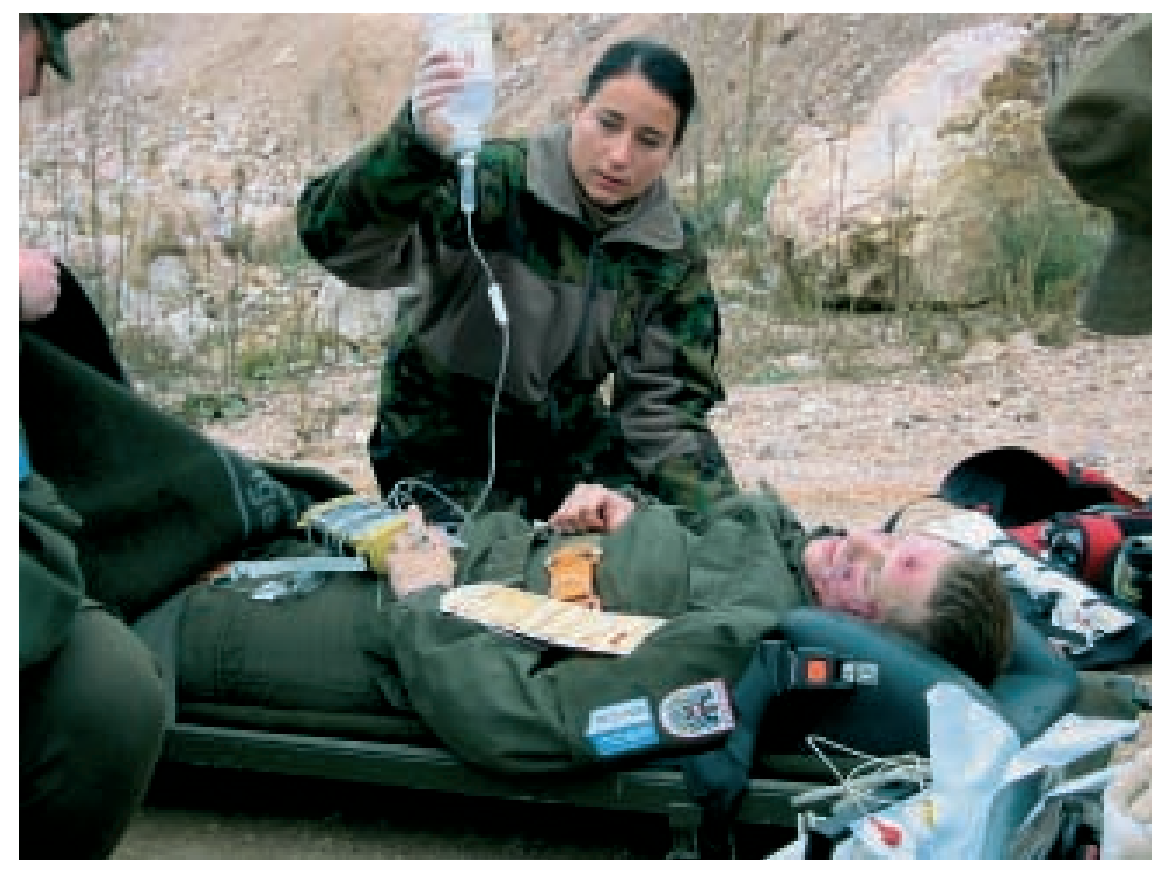

Romandie stammt, kennt sein Repertoire an Fachausdrücken natürlich am besten auf französisch. Die Österreicher mit ihren zahlreichen Dialekten sind für die Schweizer nicht immer leicht zu verstehen, das «Schwizerdütsch» bereitet wiederum den deutschen und österreichischen Kameraden Mühe. Nicht nur sprachlich bestehen Hindernisse. Während der Übung wurde deutlich, dass je nach Nation bei der Nothilfe verschiedene Handgriffe üblich sind. Dann heisst es, sich schnell untereinander einig zu werden, wie zum Beispiel ein Patient vom Unfallort auf die Bahre umgelagert werden soll.

Die Feuerwehrmänner beurteilen die beiden Unfallsituationen als durchaus praxisnah. Sie haben die Zusammenarbeit mit dem Rettungsteam als speditiv und kompetent erfahren. Auch das Feedback der «Verletzten» ist positiv: Sie haben sich gut aufgehoben gefühlt und die Betreuung als professionell empfunden.

Die Übung wird als Erfolg gewertet, einige Abläufe müssen jedoch optimiert werden. Darin sind sich alle einig. Klar ist auch, dass die Übung die Realität nicht ersetzen kann. Der Stress, die Macht der Bilder und die Eindrücke sind im Ernstfall um ein Vielfaches grösser; starke Nerven und kühle Köpfe sind dann noch viel mehr gefragt. Auch die Bergungszeit dauert bedeutend länger. Bei der Reanimation, beim Stützen oder Tragen von Patientinnen und Patienten ist je nach Szenario auch körperliche Ausdauer gefragt.

Anstelle von praktischen Übungen kann Medical Evacuation auch in Theorieblöcken trainiert werden. Konzepte und Checklisten helfen, das Gelernte abrufbereit zu halten. Eines jedoch bleibt nach dem Debriefing bei allen hängen: Man kann kaum genug üben, um für den Ernstfall gewappnet zu sein. 